$$
\lambda_{i}=\bar{u}_{i}\left(\sum_{k} f_{i k} \theta_{i k}\right)^{-1}
$$

which is equal to the definition, Eq. (7).

Numerical differences between Kennard's formula and $\mathrm{Eq}$. (5) are small, unless large differences in particle mass are involved. Consider a two-component mixture with $M_{i}>M_{k}$. Then $\lambda_{i}$ is larger than the mean free path but $\lambda_{k}$ is smaller, and the two tend to compensate one another in the summation, Eq. (6). For example, mass differences by a factor of 2 are involved in dissociating air, and estimates of viscosity ${ }^{4}$ using Kennard's mean free path for $\lambda_{i}$ are changed only a few percent when corrected according to Eq. (7). However, in the case of ionized air, the effect of collisions with electrons is vanishingly small when evaluating atom or ion mean free paths, because of the large differences in mass of the colliding particles. This was accounted for in reference 4 by neglecting such collisions entirely.

The linearized formula for viscosity coefficients can only be expected to yield approximate results, of course, since the off-diagonal matrix elements are not exactly zero, and higher-order terms in Eq. (1) do not vanish. For purposes of practical applications, the simplicity of the linearized expressions is a great advantage. According to the empirical results of Buddenberg and Wilke, ${ }^{5}$ it appears that the effects of higher-order terms can be accounted for by adjusting the coefficients in the summation of Eq. (7). For example, at ordinary temperatures and where normal, nonreactive interparticle potentials are involved, good results for the viscosities of gas mixtures are obtained if each term of the summation with $k \neq i, \mathrm{Eq}$. (7), is multiplied by a factor of about 0.69 .

1 E. H. Kennard, Kinetic Theory of Gases (McGraw-Hill Company, Inc., New York, 1938), p. 160.

2 J. O. Hirschfelder, C. F. Curtiss, and R. B. Bird, Molecular Theory of Gases and Liquids (John Wiley \& Sons, Inc., New York 1954), p. 532.

${ }^{3}$ W. E. Meador, Jr. (private communication).

4 C. F. Hansen, "Approximations for the thermodynamic and transport properties of high-temperature air," NASA Tech. Rept: 50 (1959).

${ }^{5}$ J. W. Buddenberg and C. R. Wilke, Ind. Eng. Chem. 41, 1345 (1949).

\section{Magnetically Driven Cylindrical Shock Waves}

H. W. LiEpmann and G. Vlases

California Institute of Technology, Pasadena, California (Received April 6, 1961)

$\mathrm{N}$ EARLY every experiment on shock-wave propagation uses plane waves. Cylindrical and spherical waves are more difficult to produce, usually decay fast and do not offer any.particular

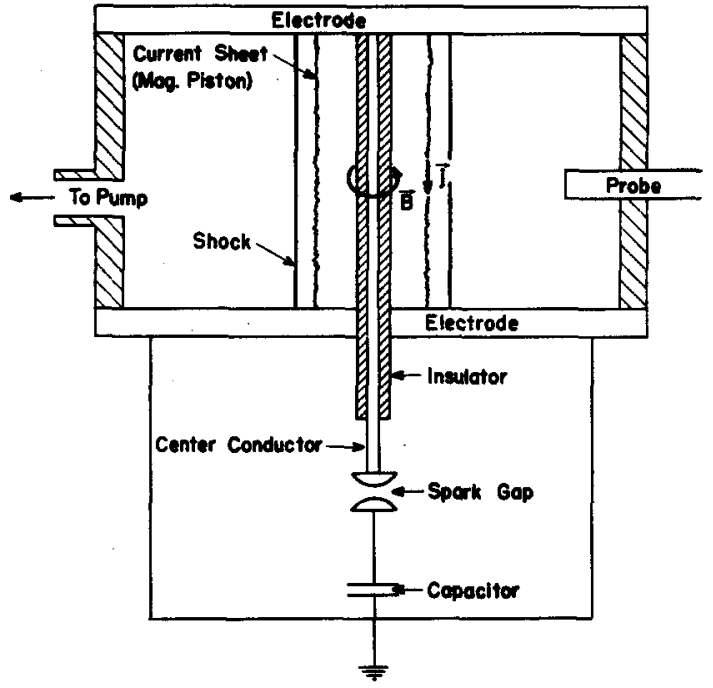

FIG. 1. Schematic diagram of apparatus.

advantages. In magneto-fluid dynamics, however, it is possible to produce cylindrical waves easily and the axisymmetric geometry is a natural choice to study motion across magnetic field lines. Furthermore, the shocks of interest are very strong and hence one can make full use of Hayes' hypersonic similitude $e^{1}$ and relate the unsteady, axisymmetric flow uniquely to an equivalent problem in steady hypersonic flow, which is often much more difficult to investigate experimentally. In the present experiments a strong cylindrical shock wave is produced by the Lorentz force acting on the outer element of a coaxial conductor made up of a center wire and the return circuit through the gas (Fig. 1). Thus in an ideally conducting gas a "contact surface" with radius $r_{c}$ is formed on which the magnetic and gas pressures $p_{c}$ are equal, i. e.,

$$
p_{c}=\mu J^{2} / 8 \pi^{2} r_{c}^{2}
$$

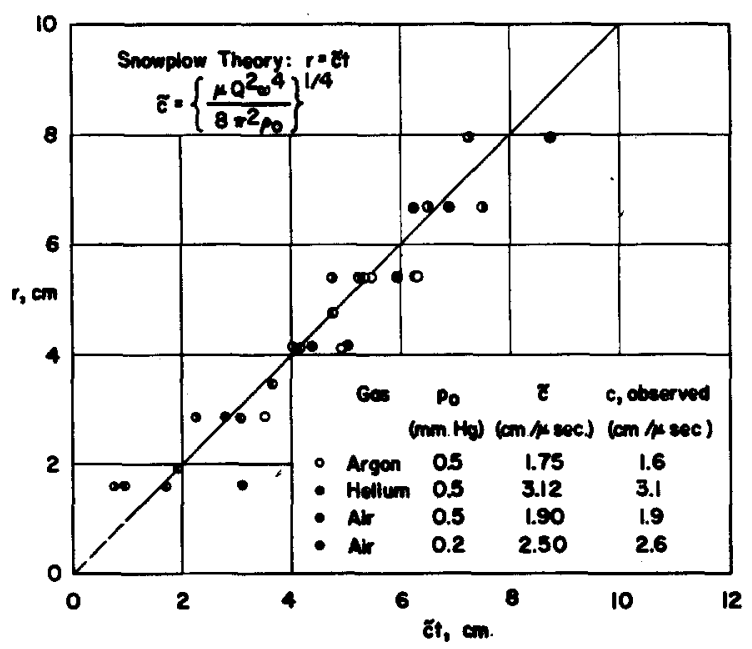

Fig. 2. Summary of pressure-front measurements. 
A given current $J(t)$ corresponds to hypersonic flow past a certain ogive $r_{c}(x)$ on which the surface pressure is given by $p_{c}(x)$. In particular $J \sim t$ corresponds to flow past a simple cone and hence $p_{c}=$ const, $r_{c}=w t$, and the shock wave radius $r=c t$ with $c / w$ depending only on the gas properties. ${ }^{2}$ The pressure $p$ behind the strong shock is given by $p \doteq \rho_{0} c^{2}\left[1-\left(\rho_{0} / \rho\right)\right]$ in terms of the undisturbed density $\rho_{0}$. For the initial current from a capacitor with charge $Q, J=Q \omega^{2} t$, and we have

$$
\begin{gathered}
c=f\left(\rho_{0} / \rho\right)\left[\mu Q^{2} \omega^{4} / 8 \pi^{2} \rho_{0}\right]^{\frac{1}{4}} \\
W=g\left(\rho_{0} / \rho\right) c .
\end{gathered}
$$

Rosenbluth's snowplow theory corresponds to $f=g=1$ and, as has been shown in a recent rigorous treatment of Greifinger and Cole, ${ }^{3}$ is completely analogous to the so-called Newtonian approximation in hypersonic flow. In a real fluid $c>w, p_{c}>p, \rho_{c}>\rho$; actual values can be obtained, e. g., from Van Dyke's solution for hypersonic cone flow. ${ }^{4}$ Finite conductivity of the gas between shock and contact surface leads to a current diffusion; the ratio of diffusion length $\delta(t)$ to $r-r_{c}$ should be small to approximate ideal conditions. The ratio is easily estimated and demonstrates the importance of a high ringing frequency of the circuit. For example, in the range where $\sigma \sim T^{3}$, one finds $\delta / r-r_{c} \sim \omega^{-2}$. The experimental setup is a natural development from an exploding wire to an "exploding cylinder" and originally the outer coaxial conductor was actually formed by a vacuumdeposited metal film on the central insulator. If the gas pressure is sufficiently low, the metal film becomes irrelevant, the return current passes immediately through the gas and the setup becomes identical with the so-called "inverse pinch" which has been studied by Anderson et al. ${ }^{5}$ from a somewhat different point of view.

In the present experiments both the arrival of the shock wave and of the current front have been observed. A small piezoelectric transducer with a rise time of $0.2 \mu$ sec, developed jointly with $T$. G. Jones was used to detect the arrival of the pressure pulse; the current front was observed by means of a small search coil. Figure 2 shows a typical space/time plot of the shock wave resulting from the discharge of a 15- $\mu \mathrm{f}$ capacitor with an initial current rise of about $130 \mathrm{ka} / \mu \mathrm{sec}$ in helium, air, and argon. Evidently the similarity solution is experimentally well realized; a departure of $f$ from unity is masked by the experimental scatter.

Over the larger part of the investigated range of densities, the observed rise time of the pressure was determined by the resolution time of the instrument corresponding to a space resolution of about $3 \mathrm{~mm}$. However, in $\mathrm{He}$ at $20 \mu$, where the mean free path is approximately $5 \mathrm{~mm}$, the expected increase in rise time was actually observed. The measurements show that stable, cylindrical waves form quickly and propagate through the fluid as predicted.

The voltage output of a search coil shows a peak of width $\tau$ corresponding to a current diffusion $\delta \doteq c \tau$. The measured values of $\delta / r$ vary between 0.025 and 0.3 in rough agreement with analytical estimates. Since $r-r_{c} \doteq \rho_{0} / 2 \rho r$, with $4 \leq \rho / \rho_{0}<10$, the detailed conditions of the flow are not nearly ideal, but the measurements do show the existence of a sharp shock wave followed by a diffusing current front. It is interesting to note that the peak of the magnetic coil trace of the wave associated with the second half-cycle oscillations of the capacitor is quite sharp compared to that of the first, indicating that the residual gas behind the first outgoing wave, due to a "leaky piston" and contamination from the Teflon center insulator, has a very high conductivity.

A more complete account of the experiments including the effects of axial magnetic field will be published soon. The experiments are made possible by the Francis I. duPont Fund for Magnetohydrodynamic Research and by a fellowship of the National Science Foundation to one of us.

${ }^{1}$ W. D. Hayes, Quart. Appl. Math. 5, 105 (1947).

2 In Kulikowsky and Javorskaga's solution for supersonic flow past a magnetized cone reported by L. I. Sedov [Revs. Modern Phys. 30. 1078 (1958)] the current is azimuthal, i. e., $J$ and $B$ are interchanged compared to our setup. The fluid mechanics remain, however, the same!

${ }^{3}$ C. Greifinger and J. D. Cole, Phys. Fluids 4, 527 (1961).

${ }^{4} \mathrm{M}$. Van Dyke, NACA TN 3173 (1954)

5 O. A. Anderson, H. P. Furth, J. M. Stone, and R. E. Wright, Phys. Fluids 1, 489 (1958).

\section{Asymmetric Collapse Accelerators for Counter-Helmholtz Geometries with Internal Injection}

\author{
RALPH W. WANIEK \\ Advanced Kinetics, Inc., Costa Mesa, California \\ AND \\ Heinz Fischer \\ A ir Force Cambridge Research Laboratories, \\ Bedford, Massachusetts \\ AND \\ Sang W. LeE \\ Advanced Kinetics, Inc., Costa Mesa, California \\ (Received December 27, 1960; \\ revised manuscript received April 28, 1961)
}

THE injection of plasma into cusped fields has received increased attention as of recently., Experiments reported so far $^{3,4}$ have made use of geometries in which plasma, usually without an internal electromagnetic structure, has been injected from a field-free region into a cusped magnetic 